\title{
E-cigarette use in public places: striking the right balance
}

Linda Bauld ${ }^{1,2}$, Ann McNeill ${ }^{3,2}$, Peter Hajek ${ }^{4,2}$, John Britton ${ }^{5,2}$ and Martin Dockrell

1. Institute for Social Marketing, University of Stirling, Stirling, UK

2. UK Centre for Tobacco and Alcohol Studies, UK

3. Institute of Psychiatry, Psychology and Neuroscience, Kings College London, London, UK

4. Wolfson Institute of Preventative Medicine, Queen Mary University of London, London, UK

5. School of Medicine, University of Nottingham, Nottingham, UK

6. Public Health England, London, UK

Corresponding author:

Professor Linda Bauld, Institute for Social Marketing, Faculty of Health Sciences and Sport, University of Stirling, Stirling FK9 4LA, UK

Linda.Bauld@stir.ac.uk

+44 (0)7714213372

Accepted for publication in Tobacco Control published by BMJ Publishing Group.

\begin{abstract}
Countries have adopted different approaches to the regulation of electronic cigarettes (e-cigarettes) and other alternative nicotine delivery devices. Some jurisdictions have decided to prohibit use in all indoor workplaces and public places where smoking is not permitted. However, is this the right approach? The United Kingdom has not adopted comprehensive public places bans, for at least three reasons. The first is that the scientific evidence of harm to bystanders from exposure to ecigarette vapour is very limited and all the existing evidence suggests that the risks are far lower than that for second hand smoke. With this in mind, applying smoke-free laws to e-cigarettes is not currently warranted on health grounds. Secondly, there is growing evidence that e-cigarettes help smokers to stop, and concern that banning them indoors sends the message to smokers that these are harmful products and may deter switching. This is particularly important as surveys show that smokers increasingly believe e-cigarettes are as dangerous as tobacco, a misperception that could undermine efforts to reduce smoking rates. Thirdly, in England, the national public health agency has conducted an extensive consultation with employers and other key stakeholders. This has produced a framework to assist decision-making about which premises choose to introduce restrictions on vaping indoors. Our view is that this type of dialogue is helpful to reach consensus and to balance the public health benefits of e-cigarettes while also acknowledging concerns about their use.
\end{abstract}




\section{Introduction}

The availability, marketing and use of electronic cigarettes or nicotine vapourisers is subject to a range of regulations across different countries, varying from the prohibition of sales and use, to little or no regulation ${ }^{1}$. The most common approach adopted in countries that permit some use of ecigarettes has been to adapt existing laws or frameworks designed for tobacco products to include ecigarettes. This can include extending bans on tobacco advertising to e-cigarettes, applying the same age of sale laws or taxing e-cigarettes like tobacco ${ }^{2}$. In some jurisdictions including many Canadian provinces and US states, existing smoke free public places laws have also been amended to include e-cigarettes, so that vaping is prohibited wherever tobacco cannot be used - including enclosed public places, workplaces and some outdoor areas. But is this the right approach, and is it supported by research evidence?

In the United Kingdom, a country with 2.8 million vapers (and 9 million smokers), policy makers have taken the decision not to legislate to ban e-cigarette use in all public places and workplaces ${ }^{2}$. This is the case in all parts of the UK (Scotland, Wales, England and Northern Ireland) despite different approaches to considering the issue and different regulatory frameworks. In England and Scotland in particular, decisions have been taken by governments that extending smokefree laws to include ecigarettes is neither desirable nor necessary to protect the health of the population.

The reasons for this are complex but relate to at least three issues. First, and most importantly, is exposure to e-cigarette vapour harmful to bystanders? Secondly, what is the role of e-cigarettes in reducing smoking rates and how might public and workplace bans help or hinder this? Thirdly, what are the views of key stakeholders?

Discussion of these three issues in the UK has been informed by the state of the evidence gathered in our own country and further afield.

\section{Is e-cigarette vapour harmful to bystanders?}

Legislation prohibiting smoking in workplaces and public places was introduced in the UK and other countries on the basis of scientific evidence that second hand smoke was harmful to bystanders ${ }^{3}$. Article 8 of the Framework Convention on Tobacco Control was developed from this evidence of health harms and sets out how smokefree laws should be implemented

Studies examining the effects of passive exposure to e-cigarette vapour on bystanders were summarised in a recent Royal College of Physicians report Nicotine without smoke: tobacco harm reduction ${ }^{2}$. The report concluded that, to date, there was no evidence that passive exposure was likely to cause significant harm to bystanders. E-cigarette users exhale vapour (an aerosol) which usually contains nicotine but there is no evidence that passive exposure to this exhaled nicotine can cause harm. Exhaled nicotine can be deposited on surfaces, but at extremely low levels and no mechanism has been proposed by which these deposits could be ingested in doses that could cause physical harm.

\section{How could a ban on e-cigarettes in indoor places affect smoking?}

We believe that potential benefits accrued from permitting e-cigarettes in some workplaces and public places outweigh concerns that allowing use might 'normalise' a behaviour similar to smoking, undermining the gains that have been made by smokefree laws. There is no evidence that ecigarettes are renormalizing tobacco smoking. Regular use of e-cigarettes by never smokers, 
including children, is minimal despite misleading headlines driven by studies that only examine ever or recent use $\mathrm{e}^{4,5,6}$. The evidence shows that in the UK, US and other countries where e-cigarette use among young people have risen, levels of tobacco use have fallen. To date the fear that e-cigarettes will create a new generation of either regular nicotine users or smokers remains unfounded.

In relation to adult smokers, current evidence suggests that e-cigarettes are unlikely to be as effective for smoking cessation as the combination of intensive behavioural support and licensed pharmacotherapies offered by well-established smoking cessation services ${ }^{7}$. However, a challenge in the UK as elsewhere is that many smokers do not access these types of services. Indeed in the UK, more than 10 times as many people use e-cigarettes to stop smoking as access services. Even with moderate effectiveness, therefore, their promise for smoking cessation may be in their reach and popularity. Yet a significant barrier to uptake of these less harmful products is public perception that they are dangerous. Smokers in the UK and elsewhere increasingly believe vaping is as risky as smoking - with this misperception rising year on year. These misperceptions influence usage ${ }^{8}$. Prohibiting the use of e-cigarettes in all places where smoking is banned is likely to promulgate the misleading message that e-cigarettes are just as harmful as smoking and therefore could deter switching from smoking to vaping.

\section{What are the views of stakeholders?}

A third important consideration on indoor e-cigarette regulations is the views of key stakeholders. Employers may be concerned about any risks from vaping either to users or bystanders, and opinions on acceptability, etiquette and enforcement are also relevant. A recent study ${ }^{9}$ in Great Britain suggested that public support for prohibiting vaping in indoor public places had increased over recent years. However, this and studies from other countries found that respondents with more accurate knowledge about the relative risks of e-cigarettes, smoking and nicotine were less likely to support such a policy. Public agencies in the UK have therefore consulted on new regulations governing e-cigarettes - including the provisions in Scotland's Health (Tobacco, Nicotine etc. and Care) Act and through Public Health England's online discussion with stakeholders about a framework for the use of e-cigarettes in enclosed public places and workplaces ${ }^{10}$. The Public Health England consultation in particular proposed draft principles for policies and practice that provided the basis for feedback from public health professionals, business owners, agencies and the public. What emerged was a consensus that national legislation was unlikely to strike an appropriate balance of risks and benefits when there was no 'one size fits all' solution. The resulting framework ${ }^{11}$, launched in July 2016, contains five principles to help guide a vaping policy appropriate for individual organisations. These were: make a clear distinction between vaping and smoking; ensure policies are based on evidence of harm to bystanders; identify and manage risks of uptake by children and young people; support smokers to stop smoking and stay smokefree; and support compliance with smokefree laws and policies. This framework allows different organisations (a nursery, for example or a factory or warehouse, which are very different working environments) to make their own decisions about where and when vaping is allowed indoors or in outdoor public spaces.

\section{Conclusion}

The evidence to date does not support a policy to prohibit e-cigarette use in enclosed public places and such policies could have significant unintended consequences by sustaining the use of smoked 
tobacco. In addition, if and when vapour products with a medicinal license become available, it will be important to allow their use indoors, just as asthma inhalers, which dispense a drug and propellants into the atmosphere, can be used indoors. It remains important, however, to continue to research the impact of exposure to e-cigarette vapour on bystanders and of policies where implemented, to ensure that evidence continues to inform practice in this area.

Words: 1,190

Competing Interests: Peter Hajek has provided consultancy for and received research funding from manufacturers of stop smoking medications. The other authors have no competing interests to declare.

\section{References}

1. MMus, A. R., \& Eisenberg, M. J. Electronic cigarettes: A comparison of national regulatory approaches. Canadian Journal of Public Health 2015; 106(6), E450.

2. RCP. Nicotine without smoke: tobacco harm reduction 2016; Royal College of Physicians, London.

3. Bauld, L. The impact of smokefree legislation in England: Evidence review 2011; Department of Health, London.

http://www.dh.gov.uk/en/Publicationsandstatistics/Publications/PublicationsPolicyAndGuid ance/DH 124961

4. Moore, G, Hewitt, G, Evans, J et al. Electronic cigarette use among young people in Wales: evidence from two cross-sectional surveys. BMJ Open 2015; 5:e007072.

5. Warner, K. Frequency of e-cigarette use and cigarette smoking by American students in 2014. American Journal of Preventive Medicine 2016; 51,2, 179-184.

6. Bauld L, MacKintosh AM, Ford A and McNeill A. E-Cigarette uptake amongst UK youth: Experimentation, but little or no regular use in non-smokers. Nicotine \& Tobacco Research 2015; doi:10.1093/ntr/ntv132

7. Brown, J., Beard, E., Kotz, D., Michie, S., \& West, R. Real-world effectiveness of e-cigarettes when used to aid smoking cessation: a cross-sectional population study. Addiction 2014; 109(9), 1531-1540.

8. Brose LS, Brown J, Hitchman S, McNeill A. Perceived relative harm of electronic cigarettes over time and impact on subsequent use. A survey with 1-year and 2-year follow-ups. Drug and Alcohol Dependence 2015; Dec 1;157:106-11. doi: 10.1016/j.drugalcdep.2015.10.014. Epub 2015 Oct 22.

9. Brose LS, Partos TR, Hitchman SC, McNeill A. Support for e-cigarette policies: a survey of smokers and ex-smokers in Great Britain. Tobacco Control 2016 Jun 16. pii: tobaccocontrol2016-052987. doi: 10.1136/tobaccocontrol-2016-052987. [Epub ahead of print]

10. Public Health England. Report of PHE stakeholder 'conversation' on use of e-cigarettes in enclosed public places and workplaces. 2015.

https://www.gov.uk/government/uploads/system/uploads/attachment data/file/534585/R eport-on-PHE-conversation-on-use-of-e-cigarettes-in-public-places.pdf [Accessed September 13th 2016)

11. Public Health England. Use of e-cigarettes in public places and workplaces: advice to inform evidence-based policy making. 2016.

https://www.gov.uk/government/uploads/system/uploads/attachment data/file/534586/P HE-advice-on-use-of-e-cigarettes-in-public-places-and-workplaces.PDF [Accessed September 13th 2016] 